\title{
End stage juvenile nephronophthisis on MRI- reply to Simms and Sayer
}

\author{
Samuel Stafrace
}

Received: 21 May 2010 / Accepted: 22 May 2010/Published online: 24 July 2010

(C) Springer-Verlag 2010

Sir,

I read with interest the letter from Simms et al. [1] regarding our clinical image presentation depicting the radiological manifestations of nephronophthisis on MRI [2]. I am grateful to the authors of this letter for taking the opportunity to further highlight the importance of this pathology, its implications and the multiple associations. In this particular case, given the described clinical presentation and the neurological associations of the radiological findings in the kidneys as outlined [1, 2], an
MRI of the brain was performed on initial admission and was normal.

\section{References}

1. Simms RJ, Sayer JA (2010) End stage juvenile nephronophthisis on MRI-a potential case of Joubert's syndrome? doi:10.1007/ s00247-010-1707-6

2. Stafrace S, Khan J (2010) End stage juvenile nephronophthisis on MRI. Pediatr Radiol. doi:10.1007/s00247-009-1535-8

S. Stafrace $(\bowtie)$

Radiology Department,

Royal Aberdeen Children's Hospital,

Foresterhill Site,

Aberdeen AB25 2ZN, UK

e-mail: samuelstafrace@nhs.net 